\title{
Quiver Generalized Weyl Algebras, Skew Category Algebras and Diskew Polynomial Rings
}

\author{
V. V. Bavula
}

Received: 7 December 2016 / Revised: 3 March 2017 / Accepted: 11 March 2017 / Published online: 28 April 2017

(C) The Author(s) 2017. This article is an open access publication

\begin{abstract}
The aim of the paper is to introduce new large classes of algebras — quiver generalized Weyl algebras, skew category algebras, diskew polynomial rings and skew semi-Laurent polynomial rings.
\end{abstract}

Keywords Skew category algebra $\cdot$ Quiver generalized Weyl algebra $\cdot$ Diskew polynomial ring - Generalized Weyl algebra $\cdot$ Skew double quiver algebra $\cdot$ Double quiver groupoid

Mathematics Subject Classification $16 \mathrm{D} 30 \cdot 16 \mathrm{P} 40 \cdot 16 \mathrm{D} 25 \cdot 16 \mathrm{P} 50 \cdot 16 \mathrm{~S} 85$

\section{Introduction}

In this paper, $K$ is a commutative ring with 1 , algebra means a $K$-algebra. In general, it is not assumed that a $K$-algebra has an identity element. Module means a left module. Missing definitions can be found in [11]. The aim of the paper is to introduce new large classes of algebras - quiver generalized Weyl algebras, skew category algebras, diskew polynomial rings, skew semi-Laurent polynomial rings and the simplex generalized Weyl algebras.

\section{Skew Category Algebras}

The aim of this section is to introduce skew category algebras; to consider new interesting examples (skew tree rings, skew semi-Laurent polynomial rings, etc); to give criteria for a skew category algebra to be a left/right Noetherian algebra (Theorem 2.2, Proposition 2.3). In Sect. 3, skew category algebras are used to define the quiver generalized Weyl algebras.

Skew category algebras Let $\mathcal{C}$ be a category, $\operatorname{Ob}(\mathcal{C})$ be the set of its objects and $\operatorname{Mor}(\mathcal{C})$ be the set of its morphisms. For objects $i, j \in \operatorname{Ob}(\mathcal{C}), \mathcal{C}(i, j)=\mathcal{C}_{j i}$ is the set of morphisms $f: i \rightarrow j$, the objects $i=t(f)$ and $j=h(f)$ are called the tail and head of the morphism $f$, respectively. For each object $i \in \operatorname{Ob}(\mathcal{C}), 1_{i}=e_{i}$ is the identity morphism $i \rightarrow i$. A ring $R$ is called a $\mathcal{C}$-graded ring if $R=\bigoplus_{a \in \operatorname{Mor}(\mathcal{C})} R_{a}$ and for all $a, b \in \operatorname{Mor}(\mathcal{C})$, $R_{a} R_{b} \subseteq R_{a b}$ if $t(a)=h(b)$ and $R_{a} R_{b}=0$, otherwise.

V. V. Bavula $(\bowtie)$

Department of Pure Mathematics, University of Sheffield, Hicks Building, Sheffield S3 7RH, UK

e-mail: V.Bavula@sheffield.ac.uk 
Definition Let $\mathcal{C}$ be a category and $\sigma$ be a functor from the category $\mathcal{C}$ to the category of $K$-algebras over a commutative ring $K$ (eg, $K=\mathbb{Z}$ or $K$ is a field). So, for each object $i \in \mathrm{Ob}(\mathcal{C}), D_{i}:=\sigma(i)$ is a $K$-algebra and for each morphism $f: i \mapsto j, \sigma_{f}: D_{i} \rightarrow D_{j}$ is a $K$-algebra homomorphism, and $\sigma_{f g}=\sigma_{f} \sigma_{g}$ for all morphisms $f$ and $g$ such that $t(f)=h(g)$. The direct sum of left $K$-modules $\mathcal{C}(\sigma)=\oplus_{f \in \operatorname{Mor}(\mathcal{C})} D_{h(f)} f$, where $D_{h(f)} f$ is a free left $D_{h(f)}$-module of rank 1, is a $K$-algebra with multiplication given by the rule: For all $f, g \in \operatorname{Mor}(\mathcal{C}), a \in D_{h(f)}$ and $b \in D_{h(g)}$,

$a f \cdot b g= \begin{cases}a \sigma_{f}(b) f g & \text { if } t(f)=h(g), \\ 0 & \text { otherwise. }\end{cases}$

The $K$-algebra $\mathcal{C}(\sigma)$ is called a skew category $K$-algebra. If $K=\mathbb{Z}$, the $\mathbb{Z}$-algebra $\mathcal{C}(\sigma)$ is called a skew category ring.

By the very definition, the algebra $\mathcal{C}(\sigma)$ is a $\mathcal{C}$-graded algebra, that is $D_{h(f)} f \cdot D_{h(g)} g \subseteq D_{h(f g)} f g$ for all $f, g \in \operatorname{Mor}(\mathcal{C})$. The algebra $\mathcal{C}(\sigma)$ is also an $\mathrm{Ob}(\mathcal{C})$-graded algebra, i.e., $\mathcal{C}(\sigma)$ is a direct sum

$\mathcal{C}(\sigma)=\bigoplus_{i, j \in \operatorname{Ob}(\mathcal{C})} \mathcal{C}(\sigma)_{i j}$ where $\mathcal{C}(\sigma)_{i j}=\bigoplus_{f \in \mathcal{C}(j, i)} D_{i} f$

and for all $i, j, k, l \in \mathrm{Ob}(\mathcal{C})$,

$\mathcal{C}(\sigma)_{i j} \mathcal{C}(\sigma)_{k l} \subseteq \delta_{j k} \mathcal{C}(\sigma)_{i l}$

where $\delta_{j k}$ is the Kronecker delta. In particular, for each $i \in \mathrm{Ob}(\mathcal{C}), \mathcal{C}(\sigma)_{i i}$ is a $K$-algebra without 1 , in general. For each $i, j \in \operatorname{Ob}(\mathcal{C}), \mathcal{C}(\sigma)_{i j}$ is a $\left(\mathcal{C}(\sigma)_{i i}, \mathcal{C}(\sigma)_{j j}\right)$-bimodule.

Example Let $\mathcal{C}$ be a category that contains a single object, say 1 , and $\operatorname{Mor}(\mathcal{C})=\left\langle x_{1}, \ldots, x_{n}\right\rangle$ is a free semigroup on $n$ elements. Then the skew category algebra $\mathcal{C}(\sigma)$ denoted by $F_{n}(\sigma)=D\left\langle x_{1}, \ldots, x_{n} ; \sigma_{x_{1}}, \ldots, \sigma_{x_{n}}, \sigma_{e}\right\rangle$ is called the skew free algebra. As an abstract ring, it is generated by a ring $D=\sigma(1)$ and elements $x_{1}, \ldots, x_{n}$ subject to the defining relations: For all $d \in D$ and $i=1, \ldots, n$,

$x_{i} d=\sigma_{x_{i}}(d) x_{i}, \quad e d=\sigma_{e}(d) e$.

Notice that $\sigma_{e} \sigma_{x_{i}}=\sigma_{x_{i}} \sigma_{e}=\sigma_{x_{i}}$. If $n=1$ and $\sigma_{e}=\operatorname{id}_{D}$ then $\mathcal{C}(\sigma)=D\left[x ; \sigma_{x}\right]$ is a skew polynomial ring. If $\sigma_{e} \neq \operatorname{id}_{D}$ then $\mathcal{C}(\sigma)$ is not a skew polynomial ring since $e d=\sigma_{e}(d) e$ and, in general, $\sigma_{e}(d) e \neq d e$ for all $d \in D$ (since $\sigma_{e} \neq \mathrm{id}_{D}$ ). For example, let $D=D_{1} \times D_{2} \times D_{3}$ and $\sigma_{e}$ and $\sigma_{x}$ are the projections onto $D_{1} \times D_{2}$ and $D_{1}$, respectively. Then $e D_{3}=0$.

Example Let $\mathcal{C}$ be a category that contains a single object, say 1 , and $\operatorname{Mor}(\mathcal{C})=\left\langle x_{1}^{ \pm 1}, \ldots, x_{n}^{ \pm 1}\right\rangle$ is a free group on $n$ elements. Then the skew category algebra $\mathcal{C}(\sigma)$ denoted by $G_{n}(\sigma)=D\left\langle x_{1}^{ \pm 1}, \ldots, x_{n}^{ \pm 1} ; \sigma_{x_{1}}, \sigma_{x_{1}^{-1}}, \ldots, \sigma_{x_{n}}, \sigma_{x_{n}^{-1}}, \sigma_{e}\right\rangle$ is called the skew free group algebra. As an abstract ring, it is generated by a ring $D=\sigma(1)$ and elements $x_{1}, x_{1}^{-1}, \ldots, x_{n}, x_{n}^{-1}$, subject to the defining relations: For all $d \in D$ and $i=1, \ldots, n$,

$x_{i} d=\sigma_{x_{i}}(d) x_{i}, \quad x_{i}^{-1} d=\sigma_{x_{i}^{-1}}(d) x_{i}, \quad e d=\sigma_{e}(d) e$.

Notice that $\sigma_{e} \sigma_{x_{i}}=\sigma_{x_{i}} \sigma_{e}=\sigma_{x_{i}}$ and $\sigma_{x_{i}} \sigma_{x_{i}^{-1}}=\sigma_{x_{i}^{-1}} \sigma_{x_{i}}=\sigma_{e}$. If $n=1$ and $\sigma_{e}=\operatorname{id}_{D}$ then $\mathcal{C}(\sigma)=$ $D\left[x, x^{-1} ; \sigma_{x}, \sigma_{x}^{-1}\right]$ is a skew polynomial ring. If $\sigma_{e} \neq \operatorname{id}_{D}$ then $\mathcal{C}(\sigma)=D\left[x, x^{-1} ; \sigma_{x}, \sigma_{x^{-1}}, \sigma_{e}\right]$ is not a skew polynomial ring since $e d=\sigma_{e}(d) e$ and, in general, $\sigma_{e}(d) e \neq d e$ for all $d \in D$ (since $\sigma_{e} \neq \mathrm{id}_{D}$ ). For example, let $D=D_{1} \times D_{2} \times D_{3}$ and $\sigma_{e}$ and $\sigma_{x}$ are the projections onto $D_{1} \times D_{2}$ and $D_{1}$, respectively. Then $e D_{3}=0$.

Definition Let $\Gamma=\left(\Gamma_{0}, \Gamma_{1}\right)$ be a tree, i.e., a connected, non-oriented graph without cycles, where $\Gamma_{0}$ is the set of vertices and $\Gamma_{1}$ is the set of edges. Let $\Gamma$ be the groupoid associated with $\Gamma: \mathrm{Ob}(\Gamma)=\Gamma_{0}$, for each $i \in \mathrm{Ob}(\Gamma)$, 
$\boldsymbol{\Gamma}(i, i)=\left\{e_{i i}\right\}$, for distinct $i, j \in \mathrm{Ob}(\boldsymbol{\Gamma})$ such that $(i, j) \in \Gamma_{1}, \boldsymbol{\Gamma}(i, j)=\left\{e_{j i}\right\}$ and $\boldsymbol{\Gamma}(j, i)=\left\{e_{i j}\right\}, e_{i j} e_{j i}=e_{i i}$ and $e_{j i} e_{i j}=e_{j j}$. Let $\sigma$ be a functor from $\boldsymbol{\Gamma}$ to the category of rings. Then $\boldsymbol{\Gamma}(\sigma)$ is called the skew tree ring. We say that the functor $\sigma$ is of isomorphism type if $\sigma\left(e_{i j}\right): \sigma(i) \rightarrow \sigma(j)$ are ring isomorphism for all $(i, j) \in \Gamma_{1}$.

Example Let $\mathcal{C}$ be a category that contains a single object, say 1 , and the monoid $\mathcal{C}(1,1)$ is generated by elements $x$ and $y$ subject to the defining relation $y x=1$. The functor $\sigma$ is determine by the algebra $D=\sigma(1)$ and its three algebra endomorphisms $\sigma_{x}, \sigma_{y}$ and $\sigma_{e}$ such that $\sigma_{y} \sigma_{x}=\sigma_{e}$. The skew category algebra $\mathcal{C}(\sigma)$ is called the skew semi-Laurent polynomial ring. It is a new class of rings. Suppose, for simplicity, that $\sigma_{e}=\mathrm{id}_{D}$. Then the ring $\mathcal{C}(\sigma)$ is generated by a ring $D$ and elements $x$ and $y$ subject to the defining relations:

$y x=1, \quad x d=\sigma_{x}(d) x$ and $y d=\sigma_{y}(d) y$ for all $d \in D$.

We denote this ring by $D\left[x, y ; \sigma_{x}, \sigma_{y}\right]$. In particular, $D\left[x, y ; \tau, \tau^{-1}\right]$ where $\tau$ is an automorphism of $D$.

The ideal $\mathfrak{a}$ and the algebra $\overline{\mathcal{C}(\sigma)} \mathrm{By}(1)$, the formal sum $e=\sum_{i \in \operatorname{Ob}(\mathcal{C})} e_{i}$ determines two well-defined maps $e \cdot: \mathcal{C}(\sigma) \rightarrow \mathcal{C}(\sigma), a \mapsto e a$, and $\cdot e: \mathcal{C}(\sigma) \rightarrow \mathcal{C}(\sigma), a \mapsto a e$. Clearly, the map $\cdot e$ is the identity map id on $\mathcal{C}(\sigma)$ but the kernel $\mathfrak{a}$ of the map $e$ is equal to $\mathfrak{a}=\oplus_{f \in \operatorname{Mor}(\mathcal{C})} \mathfrak{a}_{h(f)} f$ where $\mathfrak{a}_{i}:=\operatorname{ker}\left(\sigma_{e_{i}}\right)$ and $\sigma_{e_{i}}: D_{i} \rightarrow D_{i}$ is a $K$-algebra endomorphism.

Lemma 2.1 The set $\mathfrak{a}$ is an ideal of the algebra $\mathcal{C}(\sigma)$ such that $\mathcal{C}(\sigma) \mathfrak{a}=0, \mathfrak{a} \mathcal{C}(\sigma)=\mathfrak{a}$ and $\mathfrak{a}^{2}=0$.

Proof $\mathcal{C}(\sigma) \mathfrak{a}=\mathcal{C}(\sigma) \cdot e \cdot \mathfrak{a}=0$, the rest is obvious.

The ideal $\mathfrak{a}$ is a $\mathcal{C}$-graded ideal of the algebra $\mathcal{C}(\sigma)$. Furthermore, $\mathfrak{a}=\oplus_{i, j} \in \operatorname{Ob}(\mathcal{C}) \mathfrak{a}_{i j}$ where $\mathfrak{a}_{i j}=\oplus_{f \in \mathcal{C}(j, i)} \mathfrak{a}_{i} f \subseteq$ $\mathcal{C}(\sigma)_{i j}, \mathfrak{a}_{i j} \mathfrak{a}_{k l} \subseteq \delta_{j k} \mathfrak{a}_{i l}$ for all $i, j, k, l \in \operatorname{Ob}(\mathcal{C})$. The factor algebra $\overline{\mathcal{C}(\sigma)}=\mathcal{C}(\sigma) / \mathfrak{a}=\bigoplus_{f \in \operatorname{Mor}(\mathcal{C})} \bar{D}_{h(f)} f$ is a $\mathcal{C}$-graded algebra where $\bar{D}_{i}=D_{i} / \mathfrak{a}_{i} \simeq \operatorname{im}\left(\sigma_{i}\right)$. Furthermore,

$\overline{\mathcal{C}(\sigma)}=\bigoplus_{i, j \in \mathrm{Ob}(\mathcal{C})} \overline{\mathcal{C}(\sigma)_{i j}}$ where $\overline{\mathcal{C}(\sigma)}_{i j}=\mathcal{C}(\sigma)_{i j} / \mathfrak{a}_{i j}$

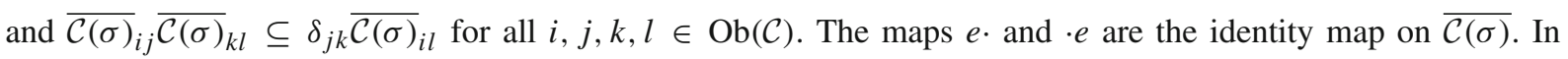
particular, the image $\bar{e}_{i}$ of the element $e_{i}$ in ${\overline{\mathcal{C}}(\sigma)_{i i}}_{i i}$ is the identity of the ring ${\overline{\mathcal{C}}(\sigma)_{i i}}_{i i}$. If $\mathfrak{a}=0$ then $e_{i}$ the identity of the algebra $\mathcal{C}(\sigma)_{i i}$. The next two results are corollaries of (3).

Theorem 2.2 (Criterion for $\mathcal{C}(\sigma)$ to be a left Noetherian algebra) The algebra $\mathcal{C}(\sigma)$ is a left Noetherian algebra iff

1. The set $\mathrm{Ob}(\mathcal{C})$ is a finite set,

2. The ideal $\mathfrak{a}$ is a finitely generated abelian group,

3. For every object $i \in \mathrm{Ob}(\mathcal{C})$, the $K$-algebra ${\overline{\mathcal{C}}(\sigma)_{i i}}_{i}$ is a left Noetherian algebra, and

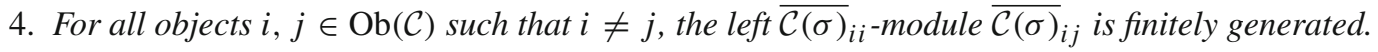

Proposition 2.3 (Criterion for $\mathcal{C}(\sigma)$ to be a right Noetherian algebra) The algebra $\mathcal{C}(\sigma)$ is a right Noetherian algebra iff

1. The set $\mathrm{Ob}(\mathcal{C})$ is a finite set,

2. For every object $i \in \mathrm{Ob}(\mathcal{C})$, the $K$-algebra $\mathcal{C}(\sigma)_{i i}$ is a right Noetherian algebra, and

3. For all objects $i, j \in \mathrm{Ob}(\mathcal{C})$ such that $i \neq j$, the right $\mathcal{C}(\sigma)_{j j}$-module $\mathcal{C}(\sigma)_{i j}$ is finitely generated.

Example Let $\mathcal{C}: 1 \stackrel{f}{\rightarrow} 2$ and the functor $\sigma$ is as follows: $\sigma(1)=K_{1}$ and $\sigma(2)=K_{2}$ are fields such that $K_{1} \subseteq K_{2}$ and $\operatorname{dim}_{K_{1}}\left(K_{2}\right)=\infty, \sigma_{e_{1}}=\mathrm{id}_{K_{1}}, \sigma_{e_{2}}=\mathrm{id}_{K_{2}}$ and $\sigma_{f}: K_{1} \rightarrow K_{2}, k \mapsto k$. Then the algebra $\mathcal{C}(\sigma)$ is isomorphic to the lower triangular matrix algebra $\left(\begin{array}{cc}K_{1} & 0 \\ K_{2} & K_{2}\end{array}\right)$. By Theorem 2.2, the algebra $\mathcal{C}(\sigma)$ is left Noetherian but not right Noetherian, by Proposition 2.3 (since $K_{2}$ is not a finitely generated right $K_{1}$-module). 


\section{Quiver Generalized Weyl Algebras}

In this section, the quiver generalized Weyl algebras (QGWAs) are introduced. Each QGWA admits two natural gradings, the object grading (see 9) and the double quiver groupoid grading (see 15). Natural classes of involutions are introduced (Proposition 3.2). An interesting subclass of QGWAs, the class of simplex generalized Weyl algebras, is introduced.

The quiver generalized Weyl algebras Let $Q=\left(Q_{0}, Q_{1}\right)$ be a quiver where $Q_{0}$ is the set of vertices and $Q_{1}$ is the set of arrows of $Q$. For vertices $i, j \in Q_{0}, Q(i, j)$ is the set of arrows from $i$ to $j$ and $|Q(i, j)|$ is the cardinality of the set $Q(i, j)$. For each arrow $a: i \rightarrow j, t(a)=i$ is the tail and $h(a)=j$ is the head of $a$. An (oriented) path from $i$ to $j$ is a formal product of arrows $a_{n} a_{n-1} \cdots a_{1}$ such that $t\left(a_{1}\right)=i, h\left(a_{1}\right)=t\left(a_{2}\right), \ldots, h\left(a_{n-1}\right)=t\left(a_{n}\right)$ and $h\left(a_{n}\right)=j$. A non-oriented path from $i$ to $j$ is a formal product of arrows $a_{n} a_{n-1} \cdots a_{1}$ such that $i \in\left\{t\left(a_{1}\right), h\left(a_{1}\right)\right\}$, $j \in\left\{t\left(a_{n}\right), h\left(a_{n}\right)\right\}$ and $\left\{h\left(a_{i}\right), t\left(a_{i}\right)\right\} \cap\left\{h\left(a_{i+1}\right), t\left(a_{i+1}\right)\right\} \neq \emptyset$ for $i=1, \ldots, n-1$. A quiver is called connected if every two distinct vertices can be connected by a non-oriented path. To be connected is an equivalence relation on the set of vertices. The set of vertices is a disjoint union of its connected components, $Q_{0}=\bigsqcup_{c \in C} Q_{0, c}$. Then the quiver $Q=\bigsqcup_{c \in C} Q_{c}$ is a disjoint union of its connected components $Q_{c}=\left(Q_{0, c}, Q_{1, c}\right)$ where $Q_{1, c}$ is the set of all arrows between vertices in $Q_{0, c}$.

Each quiver $Q$ determines the (quiver) category $Q$ where $\operatorname{Ob}(Q)=Q_{0}$ and the set of morphisms from $i$ to $j$, $Q_{j i}$, contains all oriented paths from $i$ to $j$ including the identity (the empty path) $1_{i}$ from $i \rightarrow i$ provided $i=j$. The quiver $Q$ can be seen as a set of generators of its quiver category.

The double quiver $\bar{Q}=\left(\bar{Q}_{0}, \bar{Q}_{1}\right)$ of the quiver $Q=\left(Q_{0}, Q_{1}\right)$ is a quiver such that $\bar{Q}_{0}=Q_{0}$ and for each arrow $a: i \rightarrow j$ an opposite arrow $\bar{a}: j \rightarrow i$ is added. So, $\bar{Q}_{1}=Q_{1} \bigsqcup \bar{Q}_{1}$ where $\bar{Q}_{1}=\left\{\bar{a} \mid a \in Q_{1}\right\}$, the set of opposite arrows. Let $\sigma$ be a functor from the quiver category $\bar{Q}$ to the category of $K$-algebras. For simplicity reason we assume that $\sigma\left(1_{i}\right)=\mathrm{id}_{\sigma(i)}$ for all $i \in Q_{0}$. So, for each object $i \in Q_{0}, D_{i}:=\sigma(i)$ is a $K$-algebra and for each arrow $x: i \rightarrow j, \sigma_{x}: D_{i} \rightarrow D_{j}$ is a $K$-algebra homomorphism such that $\sigma_{x y}=\sigma_{x} \sigma_{y}$ for all morphisms $x$ and $y$ such that $t(x)=h(y)$.

Definition The skew category algebra $\bar{Q}(\sigma)$ is called the skew double quiver algebra. Suppose that for each arrow $x \in Q_{1}$, there is an element $a_{x} \in D_{t(x)}$ such that

$\sigma_{\bar{x}} \sigma_{x}\left(a_{x}\right)=a_{x}, a_{x} d=\sigma_{\bar{x}} \sigma_{x}(d) a_{x}$ (for $\left.d \in D_{t(x)}\right)$ and $\sigma_{x}\left(a_{x}\right) d=\sigma_{x} \sigma_{\bar{x}}(d) \sigma_{x}\left(a_{x}\right)$ (for $\left.d \in D_{h(x)}\right)$.

Then the factor algebra (where $\left.a:=\left(a_{x}\right)_{x \in Q_{0}}\right)$

$\bar{Q}(\sigma, a):=\bar{Q}(\sigma) /\left(\bar{x} x-a_{x}, \quad x \bar{x}-\sigma_{x}\left(a_{x}\right) \mid x \in Q_{1}\right)$

is called the quiver generalized Weyl algebra (QGWA). So, as an abstract algebra, the QGWA $\bar{Q}(\sigma, a)$ is generated by the direct sum of rings $D=\oplus_{i \in Q_{0}} D_{i}$ subject to the defining relations:

$x d=\sigma_{x}(d) x \quad\left(\right.$ for $\left.d \in D_{t(x)}\right), \quad \bar{x} d=\sigma_{\bar{x}}(d) \bar{x}$ (for $\left.d \in D_{h(x)}\right), \quad \bar{x} x=a_{x}$ and $x \bar{x}=\sigma_{x}\left(a_{x}\right)$.

The QGWA $\bar{Q}(\sigma, a)$ is also denoted by $D\left[x, \bar{x} ; \sigma_{x}, \sigma_{\bar{x}}, a\right]$. Let $a_{\bar{x}}:=\sigma_{x}\left(a_{x}\right)$ for all elements $x \in \bar{Q}_{1}$. Then the equalities in (4) and (6) take a more symmetrical form:

$\sigma_{\bar{x}}\left(a_{\bar{x}}\right)=a_{x}, a_{x} d=\sigma_{\bar{x}} \sigma_{x}(d) a_{x}$ (for $\left.d \in D_{t(x)}\right)$ and $a_{\bar{x}} d=\sigma_{x} \sigma_{\bar{x}}(d) a_{\bar{x}}$ (for $d \in D_{h(x)}$ ).

$x d=\sigma_{x}(d) x \quad$ (for $\left.d \in D_{t(x)}\right), \quad \bar{x} d=\sigma_{\bar{x}}(d) \bar{x}$ (for $\left.d \in D_{h(x)}\right), \quad \bar{x} x=a_{x}$ and $x \bar{x}=a_{\bar{x}}$.

By the very definition, the ideal $\mathcal{R}=\left(\bar{x} x-a_{x}, \quad x \bar{x}-\sigma_{x}\left(a_{x}\right) \mid x \in Q_{1}\right)$ in $\bar{Q}(\sigma)$ of the defining relations of the QGWA $A=\bar{Q}(\sigma, a)$ is an $\operatorname{Ob}(\bar{Q})$-graded ideal $\mathcal{R}=\oplus_{i, j \in \mathrm{Ob}(\bar{Q})} \mathcal{R}_{i j}$ where $\mathcal{R}_{i j}=\mathcal{R} \cap \bar{Q}(\sigma)_{i j}$. So, the QGWA $A=\bar{Q}(\sigma, a)$ is an $\mathrm{Ob}(\bar{Q})$-graded algebra 
$A=\bigoplus_{i, j \in \mathrm{Ob}(\bar{Q})} A_{i j}$ where $A_{i j}=\bar{Q}(\sigma)_{i j} / \mathcal{R}_{i j}$

and for all $i, j, k, l \in \mathrm{Ob}(\bar{Q}), A_{i j} A_{k l} \subseteq \delta_{j k} A_{i l}$. For each $i \in \mathrm{Ob}(\bar{Q}), A_{i i}$ is an algebra. For $i, j \in \mathrm{Ob}(\bar{Q}), A_{i j}$ is an $\left(A_{i i}, A_{j j}\right)$-bimodule.

Definition Let $Q$ be a quiver with a single object, say 1 , and a single loop $x: 1 \rightarrow 1$. The functor $\sigma$ from the double quiver $\bar{Q}$ to the category of $K$-algebras is uniquely determined by the ring $D=\sigma(1)$ and two of its $K$-algebra endomorphisms $\sigma_{x}$ and $\sigma_{\bar{x}}$ (we assume that $\sigma\left(1_{1}\right)=\mathrm{id}_{D}$ ). Then the QGWA $A=\bar{Q}(\sigma, a)$ is generated by the ring $D$ and the elements $x, \bar{x}$ subject to the defining relations: For all elements $d \in D$,

$x d=\sigma_{x}(d) x, \quad \bar{x} d=\sigma_{\bar{x}}(d) \bar{x}, \quad \bar{x} x=a$ and $x \bar{x}=\sigma_{x}(a)$,

provided

$\sigma_{\bar{x}} \sigma_{x}(a)=a, a d=\sigma_{\bar{x}} \sigma_{x}(d) a$ and $\sigma_{x}(a) d=\sigma_{x} \sigma_{\bar{x}}(d) \sigma_{x}(a)$.

The algebra $A$ is called the generalized Weyl algebra (GWA) of rank 1 and is denoted $D\left[x, \bar{x} ; \sigma_{x}, \sigma_{\bar{x}}, a\right]$ or $D[x, y ; \sigma, \tau, a]$ where $y=\bar{x}, \sigma=\sigma_{x}$ and $\tau=\sigma_{\bar{x}}$. This definition of the GWA is an extension of the (classical) GWA, see Sect. 4 for details.

Definition The functor $\sigma$ is called of isomorphism type if $\sigma_{x}$ is an isomorphism for all $x \in Q_{1} \amalg \bar{Q}_{1}$. The functor $\sigma$ is called of invertible type if $\sigma_{\bar{x}}=\sigma_{x}^{-1}$ for all $x \in Q_{1}$ (then necessarily $\sigma_{x}=\sigma_{\bar{x}}^{-1}, a_{x} \in Z\left(D_{t(x)}\right)$ and $a_{\bar{x}} \in Z\left(D_{t(\bar{x})}\right)$ for all $x \in Q_{1}$ where $Z(R)$ is the centre of a ring $R$ ). If $\sigma$ is of invertible type then it is of isomorphism type but not vice versa, in general. We say that a QGWA $\bar{Q}(\sigma, a)$ is of isomorphism/invertible type if the functor $\sigma$ is so.

Remark A QGWA $\bar{Q}(\sigma, a)$ of invertible type is uniquely determined by the rings $\left\{D_{i}\right\}_{i \in Q_{0}}$, the isomorphisms $\left\{\sigma_{x}\right\}_{x \in Q_{1}}$ and the central elements $\left\{a_{x} \in Z\left(D_{t(x)}\right)\right\}_{x \in Q_{1}}$ that can be chosen in an arbitrary fashion since the relations (4) are automatically satisfied.

The $(x, \bar{x})$-symmetry of a QGWA Let $A=\bar{Q}(\sigma, a)=D\left[x, \bar{x} ; \sigma_{x}, \sigma_{\bar{x}}, a\right]$ be a QGWA. We define $a_{\bar{x}}:=\bar{a}_{x}:=$ $\sigma_{x}\left(a_{x}\right)$. By applying the homomorphism $\sigma_{x}$ to the equality $\sigma_{\bar{x}} \sigma_{x}\left(a_{x}\right)=a_{x}$ (i.e., $\sigma_{\bar{x}}\left(a_{\bar{x}}\right)=a_{x}$ ) we obtain the equality $\sigma_{x} \sigma_{\bar{x}}\left(a_{\bar{x}}\right)=a_{\bar{x}}$. So, the equalities in (4) imply the equalities

$\sigma_{x} \sigma_{\bar{x}}\left(a_{\bar{x}}\right)=a_{\bar{x}}, a_{\bar{x}} d=\sigma_{x} \sigma_{\bar{x}}(d) a_{\bar{x}}\left(\right.$ for $\left.d \in D_{t(\bar{x})}\right)$ and $\sigma_{\bar{x}}\left(a_{\bar{x}}\right) d=\sigma_{\bar{x}} \sigma_{x}(d) \sigma_{\bar{x}}\left(a_{\bar{x}}\right)\left(\right.$ for $\left.d \in D_{h(\bar{x})}\right)$,

where the last two equalities above coincide with the last two equalities in (4) but written in the opposite order. So, we have the QGWA $D\left[\bar{x}, x ; \sigma_{\bar{x}}, \sigma_{x}, \bar{a}\right]$ where $\bar{a}:=\sigma_{x}(a):=\left(\sigma_{x}\left(a_{x}\right)\right)=\left(\bar{a}_{x}\right)$ and

$D\left[x, \bar{x} ; \sigma_{x}, \sigma_{\bar{x}}, a\right]=D\left[\bar{x}, x ; \sigma_{\bar{x}}, \sigma_{x}, \bar{a}\right]$.

When we say the $(x, \bar{x})$-symmetry of a QGWA we mean the equality above.

The double quiver groupoid grading of a QGWA Let $\mathcal{C}$ be a category. An element $a \in \mathcal{C}(i, j)$ is called a locally invertible element or a local unit if there exists element $a^{-1} \in \mathcal{C}(j, i)$ such that $a^{-1} a=1_{i}$ and $a a^{-1}=1_{j}$. The element $a^{-1}$ is unique and is called a local inverse of $a$. By the uniqueness of the local inverse, $\left(a^{-1}\right)^{-1}=a$. If the category $\mathcal{C}$ contains a single object, say $i$, then the concepts of local unit and local inverse coincide with the concepts of unit and inverse in the monoid $\mathcal{C}(i, i)$. A category $\mathcal{C}$ is called a groupoid if all its morphisms are local units.

Let $Q$ be a quiver, $\bar{Q}$ be the double quiver of $Q$ and $\bar{Q}$ be the double quiver category associated with $\bar{Q}$. The factor category 
$G(\bar{Q})=\bar{Q} /\left(\bar{x} x-1_{t(x)}, x \bar{x}-1_{h(x)} \mid x \in Q_{1}\right)$

is a groupoid which is called the double quiver groupoid, $\mathrm{Ob}(G(\bar{Q}))=\mathrm{Ob}(\bar{Q})=Q_{0}$. Every morphism $a: i \rightarrow j$ in $G(\bar{Q})$ is a unique product $a_{n} a_{n-1} \cdots a_{1}$ where each product $a_{i+1} a_{i}$ not of the type $\bar{x} x$ or $x \bar{x}$ for some $x \in Q_{1}$. This product is called the canonical form of $a$ and the number $l(a)=n$ is called the length of $A$. So, on the level of sets we have inclusion $G(\bar{Q}) \subseteq \bar{Q}$. For all elements $f, g \in G(\bar{Q})$ such that $t(f)=h(g), l(f g) \leq l(f)+l(g)$.

Example If the category $Q=Q_{n}$ contains a single object and $n$ loops $x_{1}, \ldots, x_{n}$ then $G\left(\bar{Q}_{n}\right)$ is a free group on $n$ generators $x_{1}, \ldots, x_{n}$. If $n=1$ then $G\left(\bar{Q}_{1}\right)=\mathbb{Z}$.

Let $\mathcal{C}$ be a category. An involution $*$ on $\mathcal{C}$ is two bijections $*: \operatorname{Ob}(\mathcal{C}) \rightarrow \operatorname{Ob}(\mathcal{C}), i \mapsto i^{*}=i$, and $*: \operatorname{Mor}(\mathcal{C}) \mapsto$ $\operatorname{Mor}(\mathcal{C}), x \mapsto x^{*}$, such that $1_{i}^{*}=1_{i}$ for all $i \in \operatorname{Ob}(\mathcal{C}), \mathcal{C}(i, j)^{*}=\mathcal{C}(j, i),(x y)^{*}=y^{*} x^{*}$ and $x^{* *}=x$ for all $x, y \in \operatorname{Mor}(\mathcal{C})$. So, an involution on a category is an anti-isomorphism of order 2 that reverses the arrows and acts trivially on objects.

Let $Q$ be a quiver. The double quiver category $\bar{Q}$ admits the involution $*: x^{*}=\bar{x}$ and $\bar{x}^{*}=x$ for all $x \in Q_{1}$. The involution respects the ideal of the defining relations of the groupoid $G(\bar{Q})$. So, the groupoid $G(\bar{Q})$ admits the induced involution $*$ which is, in fact, equal the operation of taking the local inverse, $a^{*}=a^{-1}$. The QGWA $A=\bar{Q}(\sigma, a)$ is a $G(\bar{Q})$-graded algebra

$A=\bar{Q}(\sigma, a)=\bigoplus_{f \in G(\bar{Q})} A_{f}$ where $A_{f}=D_{h(f)} f$

is a free left $D_{h(f)}$-module of rank 1 (Lemma 3.1) and $A_{f} A_{g} \subseteq A_{f g}$ for all $f, g \in G(\bar{Q})$ such that $t(f)=h(g)$. Each element $a$ of the algebra $A$ is a unique finite sum $a=\sum_{f \in G(\bar{Q})} a_{f} f$ where $a_{f} \in D_{h(f)}$ and the multiplication in $A$ is given by the rule

$a f \cdot b g= \begin{cases}a \sigma_{f}(b) f g & \text { if } t(f)=h(g), l(f g)=l(f)+l(g), \\ a \sigma_{f}(b)(f, g) f g & \text { if } t(f)=h(g), l(f g)<l(f)+l(g), \\ 0 & \text { if } t(f) \neq h(g) .\end{cases}$

where $f g$ is a product of elements in the groupoid $g(\bar{Q})$ and the element $(f, g) \in D_{h(f g)}$ is defined as follows: if $t(f)=h(g)$ and $l(f g)<l(f)+l(g)$ then there is a (unique) element $h=h_{1} \cdots h_{n}$ (where $h_{i} \in \bar{Q}_{1}=Q_{1} \amalg \bar{Q}_{1}$ ) of longest length such that $f=f_{1} h$ and $g=h^{*} g$ (recall that $\left.G(\bar{Q}) \subseteq \bar{Q}\right)$. The element $h:=o(f, g)$ is called the $(f, g)$-overlap. Then

$(f, g)=\sigma_{f_{1}}\left(\left(h, h^{*}\right)\right)$ and $\left(h, h^{*}\right)=\sigma_{h_{1} \cdots h_{n-1}}\left(a_{h_{n}}\right) \sigma_{h_{1} \cdots h_{n-2}}\left(a_{h_{n-1}}\right) \cdots \sigma_{h_{1}}\left(a_{h_{2}}\right) a_{h_{1}}$

where $a_{\bar{x}}:=\sigma_{x}\left(a_{x}\right)$ for all $x \in Q_{1}$. For all elements $f \in G(\bar{Q})$,

$\left(f, f^{*}\right)=\sigma_{f}\left(\left(f^{*}, f\right)\right)$

since $\left(f^{*}, f\right) f=f f^{*} f=f\left(f^{*}, f\right)=\sigma_{f}\left(\left(f^{*}, f\right)\right)$.

Lemma 3.1 Let $A=\bar{Q}(\sigma, a)$ be a $Q G W A$. Then, for each element $f \in G(\bar{Q}), A_{f}=D_{h(f)} f$ is a free left $D_{h(f)}$-module of rank 1 , see (15).

Proof Let $A^{\prime}=\bigoplus_{f \in G(\bar{Q})} A_{f}^{\prime}$ where $A_{f}^{\prime}=D_{h(f)} f$ is a free left $D_{h(f)}$-module of rank 1 . The direct sum $A^{\prime}$ is a left $D$-module (recall that $D=\oplus_{i \in Q_{0}} D_{i}$ is the direct sum of rings) where $d_{i} \cdot d_{j} f=\delta_{i j} d_{i} d_{j} f$ for all elements $d_{i} \in D_{i}$ and $d_{j} \in D_{j}$ where $j=h(f)$. Using the defining relations (6) of the algebra $A$ it is easy to check that $A^{\prime}$ is a left $A$-module where the action of generators are defined by the rule (where $d \in D_{h(f)}$ ): 
$x \cdot d f=\left\{\begin{array}{ll}\sigma_{x}(d) x f & \text { if } t(x)=h(f), l(x f)=l(f)+1, \\ \sigma_{x}(d)(x, \bar{x}) f_{1} & \text { if } t(x)=h(f), f=\bar{x} f_{1}, \\ 0 & \text { if } t(x) \neq h(f),\end{array} \bar{x} \cdot d f= \begin{cases}\sigma_{\bar{x}}(d) \bar{x} f & \text { if } t(\bar{x})=h(f), l(\bar{x} f)=l(f)+1, \\ \sigma_{\bar{x}}(d)(\bar{x}, x) f_{2} & \text { if } t(\bar{x})=h(f), f=x f_{2}, \\ 0 & \text { if } t(\bar{x}) \neq h(f) .\end{cases}\right.$

Then the lemma follows.

Involutions on QGWAs An anti-isomorphism $*$ of a ring $R\left((a b)^{*}=b^{*} a^{*}\right.$ for all elements $\left.a, b \in R\right)$ is called an involution if $a^{* *}=a$ for all elements $a \in R$. If $R$ is a $K$-algebra and the involution $*$ is a $K$-linear map then the involution $*$ is called an algebra involution or a $K$-algebra involution.

Proposition 3.2 Let $A=\bar{Q}(\sigma, a)=D\left[x, \bar{x} ; \sigma_{x}, \sigma_{\bar{x}}, a\right]$ be a $Q G W A$. Suppose that $\sigma$ is a functor from the category $\bar{Q}$ to the category of algebras with involution such that $\sigma_{x} * \sigma_{\bar{x}}=*, \sigma_{\bar{x}} * \sigma_{x}=*, a^{*}=a$ and $(\bar{a})^{*}=\bar{a}$ (i.e., $a_{x}^{*}=a_{x}$ and $a_{\bar{x}}^{*}=a_{\bar{x}}$ for all $x \in Q_{1}$, respectively). Then the involution $*$ of the algebra $D$ can be extended to an involution $*$ of the algebra $A$ by the rule $x^{*}=\bar{x}$ and $\bar{x}^{*}=x$ for all $x \in Q_{1}$.

Proof First we show that $*$ respects the equalities (4):

(a) For all $x \in Q_{1}$ and $d \in D_{\sigma(t(x))}, a_{x} d=\sigma_{\bar{x}} \sigma_{x}(d) a_{x}:\left(\sigma_{\bar{x}} \sigma_{x}(d) a_{x}\right)^{*}=a_{x}^{*} \cdot * \sigma_{\bar{x}} \sigma_{x}(d)=a_{x} \cdot * \sigma_{\bar{x}} \sigma_{x}(d)=$ $\sigma_{\bar{x}}\left(\sigma_{x} * \sigma_{\bar{x}}\right) \sigma_{x}(d) a_{x}=\sigma_{\bar{x}} * \sigma_{x}(d) a_{x}=d^{*} a_{x}^{*}=\left(a_{x} d\right)^{*}$.

(b) For all $x \in Q_{1}$ and $d \in D_{\sigma(h(x))}, \sigma_{x}\left(a_{x}\right) d=\sigma_{x} \sigma_{\bar{x}}(d) \sigma_{x}\left(a_{x}\right):\left(\sigma_{x} \sigma_{\bar{x}}(d) \sigma_{x}(a)\right)^{*}=\sigma_{x}\left(a_{x}\right)^{*} \cdot * \sigma_{x} \sigma_{\bar{x}}(d)=$ $\sigma_{x}\left(a_{x}\right) \cdot * \sigma_{x} \sigma_{\bar{x}}(d)=\sigma_{x}\left(\sigma_{\bar{x}} * \sigma_{x}\right) \sigma_{\bar{x}}(d) \sigma_{x}\left(a_{x}\right)=\sigma_{x} * \sigma_{\bar{x}}(d) \sigma_{x}(a)=d^{*} \sigma_{x}\left(a_{x}\right)^{*}=\left(\sigma_{x}\left(a_{x}\right) d\right)^{*}$.

Next, we show that $*$ respects the defining relations (6) of the QGWA $A$ :

(i) For all $x \in Q_{1}$ and $d \in D_{\sigma(t(x))}, x d=\sigma_{x}(d) x:\left(\sigma_{x}(d) x\right)^{*}=\bar{x} \sigma_{x}(d)^{*}=\sigma_{\bar{x}} * \sigma_{x}(d) \bar{x}=d^{*} x^{*}=(x d)^{*}$.

(ii) For all $x \in Q_{1}$ and $d \in D_{\sigma(h(x))}, \bar{x} d=\sigma_{\bar{x}}(d) \bar{x}:\left(\sigma_{\bar{x}}(d) \bar{x}\right)^{*}=x \sigma_{\bar{x}}(d)^{*}=\sigma_{x} * \sigma_{\bar{x}}(d) x=d^{*} \bar{x}=(\bar{x} d)^{*}$.

(iii) $\bar{x} x=a_{x}:(\bar{x} x)^{*}=\bar{x} x=a_{x}=a_{x}^{*}$.

(iv) $x \bar{x}=\sigma\left(a_{\bar{x}}\right):(x \bar{x})^{*}=x \bar{x}=\sigma\left(a_{\bar{x}}\right)=\sigma\left(a_{\bar{x}}\right)^{*}$.

\section{The simplex generalized Weyl algebras}

Definition Let $I$ be a set that contains at least 2 elements, let $\mathcal{I}$ be a non-empty set of 2-elements subsets of $I,\left\{D_{i}\right\}_{i \in I}$ be a set of rings with identity, $\sigma=\left\{\sigma_{i j}, \sigma_{j i} \mid\{i, j\} \in \mathcal{I}\right\}$ be a set of ring homomorphisms where $\sigma_{i j}: D_{j} \rightarrow D_{i}$ and $\sigma_{j i}: D_{i} \rightarrow D_{j}, a=\left\{a_{i j}, a_{j i} \mid\{i, j\} \in \mathcal{I}\right\}$ be a set of elements where $a_{i j} \in D_{j}$ and $a_{j i} \in D_{i}$ such that

$a_{i j} d_{j}=\sigma_{j i} \sigma_{i j}\left(d_{j}\right) a_{i j}$ and $\sigma_{i j}\left(a_{i j}\right)=a_{j i}$ for all $d_{j} \in D_{j},\{i, j\} \in \mathcal{I}$.

The simplex generalized Weyl algebra (SGWA) $A=D[x ; \sigma, a]$ is a ring generated by the direct sum of rings, $D=\oplus_{i \in I} D_{i}$, and a set of (noncommutative) variables $x=\left\{x_{i j}, x_{j i} \mid\{i, j\} \in \mathcal{I}\right\}$ that satisfy the defining relations: for all $\{i, j\} \in \mathcal{I}, d_{i} \in D_{i}$ and $d_{j} \in D_{j}$,

$x_{i j} d_{j}=\sigma_{i j}\left(d_{j}\right) x_{i j}, \quad x_{i j} x_{j i}=a_{j i}, \quad D_{k} x_{i j}=0(k \neq i)$ and $x_{i j} D_{k}=0(k \neq j)$.

Clearly, a SGWA is a QGWA.

\section{Generalized Weyl Algebras and Their Simplicity Criteria}

At the beginning of the section we recall the definition of a (classical) generalized Weyl algebra and consider examples. A (classical) generalized Weyl algebra is determined by a ring $D$, its automorphism $\sigma$ and a central element $a$ of $D$. The aim of the section is to introduce a generalization of this construction, the algebras that are obtained are also called generalized Weyl algebras. Each such algebra is determined by a ring $D$, its two ring endomorphisms $\sigma$ and $\tau$ and an element $a$ of $D$ which is not assumed to be a central element of $D$ but a left normal one. We show that each such ring $A$ is a $\mathbb{Z}$-graded ring which is a free left $D$-module (Theorem 4.1) but not a 
free right $D$-module, in general. The aim of this section is to give two simplicity criteria for GWAs (Theorem 4.2 and Theorem 4.3). The first one (Theorem 4.2) is a simplicity criterion for a GWA $A=D[x, y ; \sigma, \tau, a]$ where the elements $a$ and $\sigma(a)$ are normal in $D$. This is a mild restriction on the elements $a$ and $\sigma(a)$ that often occurs in applications. The second one (Theorem 4.3) is a simplicity criterion for GWAs in general case (no restrictions on $a$ and $\sigma(a)$ ). Their proofs are quite different.

Left and right normal elements Let $D$ be a ring. An element $a \in D$ is called a left (resp., right) normal element of $D$ if $a D \subseteq D a$ (resp., $D a \subseteq a D$ ). If $a$ is a left normal element in $D$ then the left ideal $D a$ is an ideal of $D$. Similarly, if $a$ is a right normal element in $D$ then the right ideal $a D$ is an ideal of $D$. An element $a \in D$ is normal if $a D=D a$, i.e., $a$ is left and right normal. Let $\cdot a:=\cdot a_{D}: D \rightarrow D, d \mapsto d a$, and $\mathfrak{a}:=\operatorname{ker}(\cdot a)$. In particular, $\mathfrak{a} a=0$. Similarly, let $a \cdot:=a_{D} \cdot: D \rightarrow D, d \mapsto a d$, and $\mathfrak{b}:=\operatorname{ker}(a \cdot)$. In particular, $a \mathfrak{b}=0$. If the element $a$ is left normal then $\mathfrak{b}$ is an ideal of the ring $D: a \cdot D \mathfrak{b} D \subseteq D a \mathfrak{b} D=0$. If the element $a$ is right normal then $\mathfrak{a}$ is an ideal of the ring $D: D \mathfrak{a} D \cdot a \subseteq D \mathfrak{a} a D=0$. The sets $\mathbb{L}_{a}:=\left\{d \in D \mid d a=a d^{\prime}\right.$ for some $\left.d^{\prime} \in D\right\}$ and $\mathbb{R}_{a}:=\left\{d \in D \mid a d=d^{\prime} a\right.$ for some $\left.d^{\prime} \in D\right\}$ are subrings of $D$ such that $\mathfrak{a} \subseteq \mathbb{L}_{a}$ and $\mathfrak{b} \subseteq \mathbb{R}_{a}$. Furthermore, $\mathfrak{a}$ is an ideal of $\mathbb{L}_{a}\left(\mathbb{L}_{a} \mathfrak{a} \mathbb{L}_{a} \cdot a \subseteq \mathbb{L}_{a} \mathfrak{a} a D=0\right.$, and so $\left.\mathbb{L}_{a} \mathfrak{a} \mathbb{L}_{a} \subseteq \mathfrak{a}\right)$ and $\mathfrak{b}$ is an ideal of $\mathbb{R}_{a}\left(a \cdot \mathbb{R}_{a} \mathfrak{b} \mathbb{R}_{a} \subseteq D a \mathfrak{b} \mathbb{R}_{a}=0\right)$. If $a$ is a left (resp., right) normal element of $D$ then $\mathbb{L}_{a} a=a D$ (resp., $D a=a \mathbb{R}_{a}$ ).

Suppose that $a \in D$ is a left normal element. Then, for each element $d \in D, a d=d_{l} a$ for some element $d_{l} \in \mathbb{L}_{a}$ which is unique up to adding $\mathfrak{a}\left(d_{l} a=\left(d_{l}+\mathfrak{a}\right) a\right)$. Hence, the map

$\omega_{a}: D / \mathfrak{b} \rightarrow \mathbb{L}_{a} / \mathfrak{a}, \quad d+\mathfrak{b} \mapsto d_{l}+\mathfrak{a}$,

is a ring isomorphism and we can write $a d=\omega_{a}(d) d$ for all $d \in D$. A left normal element $a$ is normal iff $\mathbb{L}_{a}=D$. If $a$ is a normal element then $\mathbb{L}_{a}=D$ and the map $\omega_{a}: D / \mathfrak{b} \rightarrow D / \mathfrak{a}, d+\mathfrak{b} \mapsto d_{l}+\mathfrak{a}$, is a ring isomorphism.

\section{Generalized Weyl algebras $D(\sigma, a)$ with central element $a$}

Definition [1-8]. Let $D$ be a ring, $\sigma$ be a ring automorphism of $D, a$ is a central element of $D$. The (classical) generalized Weyl algebra (GWA, for short) $D(\sigma, a)=D[x, y ; \sigma, a]$ is a ring generated by the ring $D$ and two elements $x$ and $y$ that are subject to the defining relations:

$x d=\sigma(d) x$ and $y d=\sigma^{-1}(d) y$ for all $d \in D, \quad y x=a$ and $x y=\sigma(a)$.

The ring $D$ is called the base ring of the GWA. The automorphism $\sigma$ and the element $a$ are called the defining automorphism and the defining element of the GWA, respectively.

It is an experimental fact that many popular algebras of small Gelfand-Kirillov dimension are GWAs (see below): the first Weyl algebra $A_{1}$ and its quantum analogue, the quantum plane, the quantum sphere, $U s l(2), U_{q} \operatorname{sl}(2)$, the Heizenberg algebra and its quantum analogues, the $2 \times 2$ quantum matrices, the Witten's and Woronowic's deformations, Noetherian down-up algebras, etc., see [1-8,12].

Examples 1 1. The (first) Weyl algebra $A_{1}=K\langle x, \partial \mid \partial x-x \partial=1\rangle$ over a ring $K$ is the GWA $K[h][x, y:=$ $\partial ; \sigma, a=h]$ with base ring $K[h]$ and its $K$-automorphism $\sigma$ defined by the rule $\sigma(h)=h-1$.

2. The quantum plane $\Lambda=K\langle x, y \mid x y=q y x\rangle$ where $q$ is a unit of $K$ is the GWA $K[h][x, y ; \sigma, a=h]$ where $\sigma(h)=q h$.

3. For $q, h=q-q^{-1} \in K=\mathbb{C}$, the algebra $U_{q}=U_{q} s l(2)$ is generated by $X, Y, H_{-}$and $H_{+}$subject to the defining relations: $H_{+} H_{-}=H_{-} H_{+}=1, \quad X H_{ \pm}=q^{ \pm 1} H_{ \pm} X, \quad Y H_{ \pm}=q^{\mp 1} H_{ \pm} Y, \quad[X, Y]=\frac{H_{+}^{2}-H_{-}^{2}}{h}$. It follows that the algebra $U_{q}$ is a GWA,

$U_{q} \simeq K\left[C, H, H^{-1}\right]\left(\sigma, a=C+\left(H^{2} /\left(q^{2}-1\right)-H^{-2} /\left(q^{-2}-1\right)\right) / 2 h\right)$,

where $\sigma(H)=q H, \sigma(C)=C$.

\section{Generalized Weyl algebras}

Definition Let $D$ be a ring, $\sigma$ and $\tau$ be ring endomorphisms of $D$, and an element $a \in D$ be such that 
$\tau \sigma(a)=a, \quad a d=\tau \sigma(d) a$ and $\sigma(a) d=\sigma \tau(d) \sigma(a)$ for all $d \in D$.

The generalized Weyl algebra (GWA) $A=D(\sigma, \tau, a)=D[x, y ; \sigma, \tau, a]$ is a ring generated by $D, x$ and $y$ subject to the defining relations:

$x d=\sigma(d) x$ and $y d=\tau(d) y$ for all $d \in D, \quad y x=a$ and $x y=\sigma(a)$.

The ring $D$ is called the base ring of the GWA $A$. The endomorphisms $\sigma, \tau$ and the element $a$ are called the defining endomorphisms and the defining element of the GWA $A$, respectively. By (22), the elements $a$ and $\sigma(a)$ are left normal in $D$.

Example Let $D=C\left[t_{1}, \ldots, t_{n} ; v_{1}, \ldots, v_{n}\right]$ be a skew polynomial ring in variables $t_{1}, \ldots, t_{n}\left(t_{i} t_{j}=t_{j} t_{i}, t_{i} c=\right.$ $v_{i}(c) t_{i}$ for all $c \in C$ ) over a ring $C, v_{1}, \ldots, v_{n}$ are commuting automorphisms of the ring $C$. Suppose that an element $u \in C$ is such that $v_{i}(u)=u_{i} u$ for some unit $u_{i}$ of $D$ for $i=1, \ldots, n\left(\mathrm{eg}, u=u_{1}=\cdots=u_{n}=1\right)$. Then for each element $\alpha=\left(\alpha_{1}, \ldots, \alpha_{n}\right) \in \mathbb{N}^{n}$, the element $a=u t^{\alpha}$ is a regular, normal element of $D$ where $t^{\alpha}=t_{1}^{\alpha_{1}} \cdots t_{n}^{\alpha_{n}}$. Then $A=D\left[x, y ; \sigma, \omega_{a} \sigma^{-1}, a\right]$ is a GWA.

A $\mathbb{Z}$-grading of a GWA The next theorem proves existence of GWAs and introduces a $\mathbb{Z}$-grading.

Theorem 4.1 The GWA $A=D[x, y ; \sigma, \tau, a]$ exists. It is a $\mathbb{Z}$-graded ring $A=\oplus_{i \in \mathbb{Z}} A_{i}$ where $A_{i}=D v_{i} \simeq{ }_{D} D$, $v_{0}=1, v_{i}=x^{i}$ and $v_{-i}=y^{i}$ for $i \geq 1$. In particular, the module ${ }_{D} A$ is free.

Simplicity criteria for generalized Weyl algebras Let $D$ be a ring and $\sigma$ be its ring endomorphism. An ideal $I$ of $D$ is called $\sigma$-stable if $\sigma(I)=I$. The ring $D$ is called a $\sigma$-simple ring iff 0 and $D$ are the only $\sigma$-stable ideals of the ring $D$. An endomorphism $\sigma$ is inner if $\sigma=\omega_{u}$ for some unit $u \in D\left(\sigma(d)=u d u^{-1}\right.$ for all $\left.d \in D\right)$. Then necessarily $\sigma$ an automorphism of $D$. The next theorem is a simplicity criterion for GWAs with normal defining elements $a$ and $\sigma(a)$. It generalizes a simplicity criterion for (classical) GWAs [9, Theorem 4.2]. The idea of the proofs of the next two theorems is to use localizations and graded/filtered techniques.

Theorem 4.2 [10] Let $A=D[x, y ; \sigma, \tau, a]$ be a GWA such that the elements $a$ and $\sigma(a)$ are right normal in $D$ (they are normal, by (22)). Then the following statement are equivalent.

1. A is a simple ring.

2. (a) The elements $a$ and $\sigma(a)$ are regular in $D$,

(b) $D$ is a $\sigma$-simple ring,

(c) for all $i \geq 1, \sigma^{i}$ is not an inner automorphism of the ring $D$, and

(d) for all $i \geq 1, D a+D \sigma^{i}(a)=D$.

3. (a) The elements $a$ and $\sigma(a)$ are regular in $D$,

(b) $D$ is a $\tau$-simple ring,

(c) for all $i \geq 1, \tau^{i}$ is not an inner automorphism of the ring $D$, and

(d) for all $i \geq 1, D \sigma(a)+D \tau^{i} \sigma(a)=D$.

If one of the equivalent conditions holds then $\sigma$ and $\tau$ are automorphisms of $D$.

Example Let $A=\Lambda\left[x, y ; \sigma=\sigma_{\alpha, \beta}, \tau=\omega_{a} \sigma^{-1}, a\right]$ be the GWA considered in Sect. 4. If $a \notin K^{*}$ then $\sigma^{i}(a) \in$ $K^{*} a$ for all $i \geq 1$, and so the condition (2d) of Theorem 4.2 does not hold and the algebra $A$ is not simple.

For a ring $D$ and its ring endomorphism $\sigma$, the subring of $D, D^{\sigma}=\{d \in D \mid \sigma(d)=d\}$, is called the ring of $\sigma$-invariants, and each element of $D^{\sigma}$ is called a $\sigma$-invariant. Every left normal, left regular element $d$ of $D$, determines a ring endomorphism of $D$ :

$\omega_{d}: D \rightarrow D, \quad d^{\prime} \mapsto \omega_{d}\left(d^{\prime}\right)$, where $d d^{\prime}=\omega_{d}\left(d^{\prime}\right) d$.

The next theorem is a simplicity criterion for GWAs. 
Theorem 4.3 [10] Let $A=D[x, y ; \sigma, \tau, a]$ be a GWA. Then the following statements are equivalent.

1. A is a simple ring.

2. (a) The elements $a$ and $\sigma(a)$ are regular in $D$,

(b) For all nonzero ideals $I$ of $D, I^{\prime}=D$ where $I^{\prime}:=I+\sum_{i \geq 1}\left(D \sigma^{i}(I)(i,-i)+D \tau^{i}(I)(-i, i)\right)$.

(c) None of the ring endomorphisms $\sigma^{n}(n \geq 1)$ of $D$ is equal to the ring endomorphism $\omega_{d}$ (see (24)) where $d$ is a $\sigma$-invariant, regular, left normal element of $D$.

3. (a) The elements $a$ and $\sigma(a)$ are regular in $D$,

(b) For all nonzero ideals $I$ of $D, I^{\prime}=D$ where $I^{\prime}:=I+\sum_{i \geq 1}\left(D \sigma^{i}(I)(i,-i)+D \tau^{i}(I)(-i, i)\right)$.

(c) None of the ring endomorphisms $\tau^{n}(n \geq 1)$ of $D$ is equal to the ring endomorphism $\omega_{d}($ see (24)) where $d$ is a $\tau$-invariant, regular, left normal element of $D$.

If one of the equivalent conditions holds then $\sigma$ and $\tau$ are monomorphisms of $D$.

\section{Generalized Weyl Algebras of Rank $n$}

The aim of this section is to introduce a new class of rings which is more general that the class of (classical) generalized Weyl algebras. The rings of the new class are also called generalized Weyl algebras. In order to distinguish these new rings from the old ones the latter are called classical GWAs.

Iterated generalized Weyl algebras The next corollary follows from Theorem 4.1 by induction on the rank $n$.

Corollary 5.1 Let $A=D\left[x_{1}, y_{1} ; \sigma_{1}, \tau_{1}, a_{1}\right] \ldots\left[x_{n}, y_{n} ; \sigma_{n}, \tau_{n}, a_{n}\right]$ be an iterated $G W A$ of rank $n$. Then $A=$ $\oplus_{\alpha \in \mathbb{Z}^{n}} D v_{\alpha}$ is a direct sum of the free left $D$-modules ${ }_{D} D v_{\alpha} \simeq D$ wherefor $\alpha=\left(\alpha_{1}, \ldots, \alpha_{n}\right), v_{\alpha}=v_{\alpha_{1}}(1) \cdots v_{\alpha_{n}}(n)$ and $v_{\alpha_{i}}(i)= \begin{cases}x_{i}^{\alpha_{i}} & \text { if } \alpha_{i} \geq 0, \\ y_{i}^{-\alpha_{i}} & \text { if } \alpha_{i}<0 .\end{cases}$

Classical generalized Weyl algebras, [1-8] Let $D$ be a ring, $\sigma=\left(\sigma_{1}, \ldots, \sigma_{n}\right)$ an $n$-tuple of commuting automorphisms of $D, a=\left(a_{1}, \ldots, a_{n}\right)$ an $n$-tuple of elements of the centre $Z(D)$ of $D$ such that $\sigma_{i}\left(a_{j}\right)=a_{j}$ for all $i \neq j$. The (classical) generalized Weyl algebra $A=D(\sigma, a)=D[x, y ; \sigma, a]$ of rank $n$ is a ring generated by $D$ and $2 n$ indeterminates $x_{1}, \ldots, x_{n}, y_{1}, \ldots, y_{n}$ subject to the defining relations:

$$
\begin{aligned}
& y_{i} x_{i}=a_{i}, \quad x_{i} y_{i}=\sigma_{i}\left(a_{i}\right), \quad x_{i} d=\sigma_{i}(d) x_{i}, \text { and } y_{i} d=\sigma_{i}^{-1}(d) y_{i} \text { for all } d \in D, \\
& {\left[x_{i}, x_{j}\right]=\left[x_{i}, y_{j}\right]=\left[y_{i}, y_{j}\right]=0, \text { for all } i \neq j,}
\end{aligned}
$$

where $[x, y]=x y-y x$. We say that $a$ and $\sigma$ are the sets of defining elements and automorphisms of the GWA $A$, respectively.

Generalized Weyl algebras Let $A$ be a ring and $\sigma$ its endomorphism. A subring $B$ of $A$ is called $\sigma$-invariant if $\sigma(B) \subseteq B$.

Definition An iterated generalized Weyl algebra $A=D\left[x_{1}, y_{1} ; \sigma_{1}, \tau_{1}, a_{1}\right] \ldots\left[x_{n}, y_{n} ; \sigma_{n}, \tau_{n}, a_{n}\right]$ is called a generalized Weyl algebra of rank $n$ if $a_{1}, \ldots, a_{n} \in D$, the ring $D$ is $\sigma_{i}$ - and $\tau_{i}$-invariant for all $i=1, \ldots, n$; and for all integers $i, j=1, \ldots, n$ such that $i>j$ :

$\sigma_{i}\left(x_{j}\right)=\lambda_{i j} x_{j}, \quad \sigma_{i}\left(y_{j}\right)=\lambda_{i j}^{\prime} y_{j}, \quad \tau_{i}\left(x_{j}\right)=\mu_{i j} x_{j}, \quad \tau_{i}\left(y_{j}\right)=\mu_{i j}^{\prime} y_{j}$,

for some elements $\lambda_{i j}, \lambda_{i j}^{\prime}, \mu_{i j}$ and $\mu_{i j}^{\prime}$ of the ring $D$. The elements $\Lambda=\left(\lambda_{i j}\right), \Lambda^{\prime}=\left(\lambda_{i j}^{\prime}\right), M=\left(\mu_{i j}\right)$ and $M^{\prime}=\left(\mu_{i j}^{\prime}\right)$ are called the defining coefficients of $A$. The $n$-tuples of endomorphisms $\sigma=\left(\sigma_{1}, \ldots, \sigma_{n}\right)$ and $\tau=\left(\tau_{1}, \ldots, \tau_{n}\right)$ are called the defining endomorphisms of $A$, and the $n$-tuples of elements $a=\left(a_{1}, \ldots, a_{n}\right)$ is called the defining elements of $A$. The GWA $A$ of rank $n$ is denoted by $A=D\left[x, y ; \sigma, \tau, \Lambda, \Lambda^{\prime}, M, M^{\prime}\right]$ where $x=\left(x_{1}, \ldots, x_{n}\right)$ and $y=\left(y_{1}, \ldots, y_{n}\right)$.

We denote by $\sigma_{i}$ and $\tau_{i}$ the restrictions $\left.\sigma_{i}\right|_{D}$ and $\left.\tau_{i}\right|_{D}$, respectively. 
An element $\Lambda=\left(\lambda_{i j}\right)$ (where $\left.1 \leq j<i \leq n\right)$ is called a lower triangular half-matrix with coefficients in $D$. The set of all such elements is denoted by $L_{n}(D)$. The next proposition describes GWAs of rank $n$ via generators and defining relations.

Proposition 5.2 [10] Let $D$ be a ring, $\sigma=\left(\sigma_{i}\right)$ and $\tau=\left(\tau_{i}\right)$ n-tuples of ring endomorphisms of $D, a=\left(a_{i}\right) \in D^{n}$, and $\Lambda=\left(\lambda_{i j}\right), \Lambda^{\prime}=\left(\lambda_{i j}^{\prime}\right), M=\left(\mu_{i j}\right), M^{\prime}=\left(\mu_{i j}^{\prime}\right) \in L_{n}(D)$ such that the following conditions hold: For all $i=1, \ldots, n$ and $d \in D$,

$\tau_{i} \sigma_{i}\left(a_{i}\right)=a_{i}, \quad a_{i} d=\tau_{i} \sigma_{i}(d) a_{i}$ and $\sigma_{i}\left(a_{i}\right) d=\sigma_{i} \tau_{i}(d) \sigma_{i}\left(a_{i}\right)$

for all $i>j$,

$$
\begin{aligned}
a_{i} & =\tau_{i}\left(\lambda_{i j}\right) \mu_{i j} \sigma_{j}\left(a_{i}\right)=\tau_{i}\left(\lambda_{i j}^{\prime}\right) \mu_{i j}^{\prime} \tau_{j}\left(a_{i}\right), \\
\sigma_{i}\left(a_{i}\right) & =\sigma_{i}\left(\mu_{i j}\right) \lambda_{i j} \sigma_{j} \sigma_{i}\left(a_{i}\right)=\sigma_{i}\left(\mu_{i j}^{\prime}\right) \lambda_{i j}^{\prime} \tau_{j} \sigma_{i}\left(a_{i}\right) ;
\end{aligned}
$$

for all $i>j$ and $d \in D$,

$$
\begin{aligned}
\lambda_{i j} \sigma_{j} \sigma_{i}(d) & =\sigma_{i} \sigma_{j}(d) \lambda_{i j} \text { and } \mu_{i j} \sigma_{j} \tau_{i}(d)=\tau_{i} \sigma_{j}(d) \mu_{i j}, \\
\lambda_{i j}^{\prime} \tau_{j} \sigma_{i}(d) & =\sigma_{i} \tau_{j}(d) \lambda_{i j}^{\prime} \text { and } \mu_{i j}^{\prime} \tau_{j} \tau_{i}(d)=\tau_{i} \tau_{j}(d) \mu_{i j}^{\prime}, \\
\sigma_{i}\left(a_{j}\right) & =\lambda_{i j}^{\prime} \tau_{j}\left(\lambda_{i j}\right) a_{j} \text { and } \tau_{i}\left(a_{j}\right)=\mu_{i j}^{\prime} \tau_{j}\left(\mu_{i j}\right) a_{j}, \\
\sigma_{i} \sigma_{j}\left(a_{j}\right) & =\lambda_{i j} \sigma_{j}\left(\lambda_{i j}^{\prime}\right) \sigma_{j}\left(a_{j}\right) \text { and } \tau_{i} \sigma_{j}\left(a_{j}\right)=\mu_{i j} \sigma_{j}\left(\mu_{i j}^{\prime}\right) \sigma_{j}\left(a_{j}\right) .
\end{aligned}
$$

for $i>j>k: \quad \lambda_{i j} \sigma_{j}\left(\lambda_{i k}\right) \lambda_{j k}=\sigma_{i}\left(\lambda_{j k}\right) \lambda_{i k} \sigma_{k}\left(\lambda_{i j}\right)$ and $\lambda_{i j} \sigma_{j}\left(\lambda_{i k}^{\prime}\right) \lambda_{j k}^{\prime}=\sigma_{i}\left(\lambda_{j k}^{\prime}\right) \lambda_{i k}^{\prime} \tau_{k}\left(\lambda_{i j}\right)$,

$$
\lambda_{i j}^{\prime} \tau_{j}\left(\lambda_{i k}\right) \mu_{j k}=\sigma_{i}\left(\mu_{j k}\right) \lambda_{i k} \sigma_{k}\left(\lambda_{i j}^{\prime}\right) \text { and } \lambda_{i j}^{\prime} \tau_{j}\left(\lambda_{i k}^{\prime}\right) \mu_{j k}^{\prime}=\sigma_{i}\left(\mu_{j k}^{\prime}\right) \lambda_{i k}^{\prime} \tau_{k}\left(\lambda_{i j}^{\prime}\right) \text {, }
$$

$$
\mu_{i j} \sigma_{j}\left(\mu_{i k}\right) \lambda_{j k}=\tau_{i}\left(\lambda_{j k}\right) \mu_{i k} \sigma_{k}\left(\mu_{i j}\right) \text { and } \mu_{i j} \sigma_{j}\left(\mu_{i k}^{\prime}\right) \lambda_{j k}^{\prime}=\tau_{i}\left(\lambda_{j k}\right) \mu_{i k}^{\prime} \tau_{k}\left(\mu_{i j}\right) \text {, }
$$

$$
\mu_{i j}^{\prime} \tau_{j}\left(\mu_{i k}\right) \mu_{j k}=\tau_{i}\left(\mu_{j k}\right) \mu_{i k} \sigma_{k}\left(\mu_{i j}^{\prime}\right) \text { and } \mu_{i j}^{\prime} \tau_{j}\left(\mu_{i k}^{\prime}\right) \mu_{j k}^{\prime}=\tau_{i}\left(\mu_{j k}^{\prime}\right) \mu_{i k}^{\prime} \tau_{k}\left(\mu_{i j}^{\prime}\right) .
$$

The GWA of rank $n, A=D\left[x, y ; \sigma, \tau, a, \Lambda, \Lambda^{\prime}, M, M^{\prime}\right]$, is a ring generated by $D, x_{1}, \ldots, x_{n}$ and $y_{1}, \ldots, y_{n}$ subject to the defining relations: For all $i=1, \ldots, n$ and $d \in D$,

$x_{i} d=\sigma_{i}(d) x_{i}, \quad y_{i} d=\tau_{i}(d) y_{i}, \quad y_{i} x_{i}=a_{i}$ and $x_{i} y_{i}=\sigma_{i}\left(a_{i}\right)$

for all $i>j$,

$x_{i} x_{j}=\lambda_{i j} x_{j} x_{i}, \quad x_{i} y_{j}=\lambda_{i j}^{\prime} y_{j} x_{i}, \quad y_{i} x_{j}=\mu_{i j} x_{j} y_{i}$ and $y_{i} y_{j}=\mu_{i j}^{\prime} y_{j} y_{i}$.

Example If $\sigma=\left(\sigma_{1}, \ldots, \sigma_{n}\right) \in \operatorname{Aut}(D)^{n}$ is an $n$-tuple of commuting automorphisms of the $\operatorname{ring} D, \tau:=\sigma^{-1}=$ $\left(\sigma_{1}^{-1}, \ldots, \sigma_{n}^{-1}\right), a=\left(a_{1}, \ldots, a_{n}\right) \in Z(D)$ and $\sigma_{i}\left(a_{j}\right)=a_{j}$ for all $i \neq j$; and $\lambda_{i j}=\lambda_{i j}^{\prime}=\mu_{i j}=\mu_{i j}^{\prime}=1$ for all $i>j$, then the GWA $A$ of rank $n$ is a classical GWA of rank $n$, that is $A=D[x, y ; \sigma, a]$.

Recall that each normal, regular element $\alpha$ of a ring $D$ determines the automorphism $\omega_{\alpha}$ of $D$ by the rule $\alpha d=\omega_{\alpha}(d) \alpha$ for all $d \in D$. The next proposition gives plenty of examples of GWAs of rank $n$.

Proposition 5.3 Let $D$ be a ring, $\theta_{1}, \ldots, \theta_{n}$ commuting automorphisms of the ring $D, \alpha_{1}, \ldots, \alpha_{n}, \beta_{1}, \ldots, \beta_{n}$ regular, normal elements of $D$. Then $A=D\left[x, y ; \sigma, \tau, a, \Lambda, \Lambda^{\prime}, M, M^{\prime}\right]$ be a $G W A$ of rank $n$ where

$$
\begin{aligned}
\sigma_{i} & =\theta_{i} \omega_{\beta_{i}}, \quad \tau_{i}=\omega_{\alpha_{i}} \theta_{i}^{-1}, \quad a_{i}=\alpha_{i} \beta_{i}, \\
\lambda_{i j} & =\theta_{i}\left(\beta_{i}\right) \theta_{i} \theta_{j}\left(\beta_{j} \beta_{i}^{-1}\right) \theta_{j}\left(\beta_{j}^{-1}\right), \quad \lambda_{i j}^{\prime}=\theta_{i}\left(\beta_{i} \alpha_{j}\right) \cdot \theta_{j}^{-1} \theta_{i}\left(\beta_{i}^{-1}\right) \alpha_{j}^{-1}, \\
\mu_{i j} & =\alpha_{i} \theta_{i}^{-1} \theta_{j}\left(\beta_{j}\right) \theta_{j}\left(\alpha_{i}^{-1} \beta_{j}^{-1}\right), \quad \mu_{i j}^{\prime}=\alpha_{i} \theta_{i}^{-1}\left(\alpha_{j}\right) \theta_{j}^{-1}\left(\alpha_{i}^{-1}\right) \alpha_{j}^{-1},
\end{aligned}
$$

provided $\lambda_{i j}, \lambda_{i j}^{\prime}, \mu_{i j}, \mu_{i j} \in D$. 
A $\mathbb{Z}^{n}$-grading of a GWA of rank $n$ By Theorem 4.1, every GWA of rank $n, A=D\left[x, y ; \sigma, \tau, a, \Lambda, \Lambda^{\prime}, M, M^{\prime}\right]$, is a $\mathbb{Z}^{n}$-graded algebra $A=\oplus_{\alpha \in \mathbb{Z}^{n}} D v_{\alpha}\left(D v_{\alpha} D v_{\beta} \subseteq D v_{\alpha+\beta}\right.$ for all elements $\left.\alpha, \beta \in \mathbb{Z}^{n}\right)$ where for $\alpha=$ $\left(\alpha_{1}, \ldots, \alpha_{n}\right) \in \mathbb{Z}^{n}, v_{\alpha}=v_{\alpha_{1}}(1) v_{\alpha_{2}}(2) \cdots v_{\alpha_{n}}(n)$ and $v_{\alpha_{i}}(i):=\left\{\begin{array}{ll}x_{i}^{\alpha_{i}} & \text { if } \alpha_{i} \geq 0, \\ y_{i}^{-\alpha_{i}} & \text { if } \alpha_{i}<0 .\end{array}\right.$. Notice that the order in the product for $v_{\alpha}$ is important and, in general, cannot be changed. Moreover, the left $D$-module $D v_{\alpha}$ is free of rank 1 . For all elements $\alpha, \beta \in \mathbb{Z}^{n}$,

$v_{\alpha} v_{\beta}=(\alpha, \beta) v_{\alpha+\beta}$

for some (explicit) elements $(\alpha, \beta) \in D$. For all elements $\alpha \in \mathbb{Z}^{n}$ and $d \in D, v_{\alpha} d=\sigma^{\alpha}(d) v_{\alpha}$ where $\sigma^{\alpha}:=$ $\sigma\left(1, \alpha_{1}\right) \cdots \sigma\left(n, \alpha_{n}\right)$ and $\sigma\left(i, \alpha_{i}\right):= \begin{cases}\sigma_{i}^{\alpha_{i}} & \text { if } \alpha_{i} \geq 0 \\ \tau_{i}^{-\alpha_{i}} & \text { if } \alpha_{i}<0\end{cases}$

\section{Diskew Polynomial Rings}

The aim of this section is to introduce a new class of rings - the diskew polynomial rings - to show that they are GWAs under a mild restriction (Theorem 6.2). To give a simplicity criterion, Theorem 6.4, for diskew polynomial rings that are GWAs satisfying the conditions of Theorem 4.2. It is a corollary of Theorem 4.2. The proofs can be found in [10].

\section{Diskew polynomial rings}

Definition Let $D$ be a ring, $\sigma$ and $\tau$ be its ring endomorphisms, $\rho$ and $b$ be elements of $D$ such that, for all $d \in D$,

$\sigma \tau(d) \rho=\rho \tau \sigma(d)$ and $\sigma \tau(d) b=b d$,

The diskew polynomial ring (DPR) $E:=D(\sigma, \tau, b, \rho):=D[x, y ; \sigma, \tau, b, \rho]$ is a ring generated by $D, x$ and $y$ subject to the defining relations:

$x d=\sigma(d) x$ and $y d=\tau(d) y$ for all $d \in D, \quad x y-\rho y x=b$.

Example The quantum plane $\Lambda=K\langle p, q \mid p q=\lambda q p\rangle$ (over a field $K$ where $\lambda \in K^{*}$ ) is a skew polynomial ring $\Lambda=K[q][p ; v]$ where $v(q)=\lambda q$. Then $E=\Lambda\left[x, y ; v^{\alpha}, v^{\beta}, \eta t^{\alpha+\beta}, \rho\right]$ is a diskew polynomial ring where $\eta, \rho \in K^{*}$ and $\alpha, \beta \in \mathbb{N}$ (see the previous example).

Theorem 6.1 The diskew polynomial ring $E=D[x, y ; \sigma, \tau, b, \rho]$ exists. It is a free left $D$-module $E=$ $\oplus_{i, j \in \mathbb{N}} D y^{i} x^{j}$ and the element $x$ is a left regular element.

\section{Diskew rings are GWAs when $\rho$ is a unit}

Theorem 6.2 Let $E=D[x, y ; \sigma, \tau, b, \rho]$ be a diskew polynomial ring. Suppose that $\rho$ is a unit in $D$. Then $x$ and $y$ are left regular elements of $E$ and the ring $E=\mathcal{D}[x, y ; \sigma, \tau, a=h]$ is a GWA with base ring $\mathcal{D}:=D[h ; \tau \sigma]$ which is a skew polynomial ring, $\sigma$ and $\tau$ are ring endomorphisms of $\mathcal{D}$ that are extensions of the ring endomorphisms $\sigma$ and $\tau$ of $D$, respectively, defined by the rule $\sigma(h)=\rho h+b$ and $\tau(h)=\tau\left(\rho^{-1}\right)(h-\tau(b))$. In particular, $\tau \sigma(h)=h$ and $\sigma \tau(h)=\omega_{\rho}(h)=\rho \tau \sigma\left(\rho^{-1}\right) h$. Furthermore, $\sigma \tau=\omega_{\rho} \tau \sigma$ in $\mathcal{D}$.

Corollary 6.3 Let $E=D[x, y ; \sigma, \tau, b, \rho]$ be a diskew polynomial ring. Suppose that $\rho$ is a unit in $D$. Then $E=\mathcal{D}\left[y, x ; \tau, \sigma, h^{\prime}:=\sigma(h)=\rho h+b\right]$ is a GWA with base ring $\mathcal{D}:=D[h ; \tau \sigma]=D\left[h^{\prime}, \sigma \tau\right]$ which is a skew polynomial ring, $\sigma$ and $\tau$ are ring endomorphism of $\mathcal{D}$ that are extensions of the ring endomorphisms $\sigma$ and $\tau$ of $D$, respectively, defined in Theorem 6.2; $\tau\left(h^{\prime}\right)=\rho^{-1}\left(h^{\prime}-b\right)$ and $\sigma\left(h^{\prime}\right)=\sigma(\rho) h^{\prime}+\sigma(b)$. In particular, $\sigma \tau\left(h^{\prime}\right)=h^{\prime}$ and $\tau \sigma\left(h^{\prime}\right)=\omega_{\rho^{-1}}\left(h^{\prime}\right)=\rho^{-1} \sigma \tau(\rho) h^{\prime}$. 
By Theorem 6.2, if $\rho \in D$ is a unit then the ring endomorphisms $\sigma$ and $\tau$ of $D$ can be extended to the ring $\mathcal{D}=D[h ; \tau \sigma]$ by the rule $\sigma(h)=\rho h+b$ and $\tau(h)=\tau\left(\rho^{-1}\right)(h-\tau(b))$. By induction on $i \geq 1$, we have the equalities where

$$
\begin{aligned}
& \sigma^{i}(h)=a_{i} h+b_{i}, \quad a_{i}=\sigma^{i-1}(\rho) \cdots \sigma(\rho) \rho \text { and } b_{i}=\sum_{1 \leq j \leq i-1} \sigma^{j}\left(a_{i-j}\right) \sigma^{j-1}(b)+\sigma^{i-1}(b), \\
& \tau^{i}(h)=a_{i}^{\prime} h+b_{i}^{\prime}, \quad a_{i}^{\prime}=\tau^{i-1}\left(\rho^{-1}\right) \cdots \tau^{2}\left(\rho^{-1}\right) \tau\left(\rho^{-1}\right), \quad b_{i}^{\prime}=-\sum_{j=1}^{i-1} \tau^{j}\left(a_{i-j}^{\prime}\right) \tau^{j}\left(\rho^{-1} b\right)-\tau^{i}\left(\rho^{-1} b\right) .
\end{aligned}
$$

In particular, for all $i \geq 1$,

$a_{i+1}=\sigma\left(a_{i}\right) \rho$ and $b_{i+1}=\sigma\left(a_{i}\right) b+\sigma\left(b_{i}\right)$,

$a_{i+1}^{\prime}=\tau\left(a_{i}^{\prime}\right) \tau\left(\rho^{-1}\right)$ and $b_{i+1}^{\prime}=-\tau\left(a_{i}^{\prime}\right) \tau\left(\rho^{-1} b\right)+\tau\left(b_{i}^{\prime}\right)$.

For example, (42) follows from $a_{i+1} h+b_{i+1}=\sigma\left(\sigma^{i}(h)\right)=\sigma\left(a_{i} h+b_{i}\right)=\sigma\left(a_{i}\right)(\rho h+b)+\sigma\left(b_{i}\right)=\sigma\left(a_{i}\right) \rho h+$ $\sigma\left(a_{i}\right) b+\sigma\left(b_{i}\right)$.

Suppose that $\rho$ is a unit. Then $\sigma \tau=\omega_{\rho} v$ where $v=\tau \sigma$, or, equivalently, $\omega_{\rho^{-1}} \sigma \tau=v$. Let $\beta:=\rho^{-1} b$. It follows that for all $d \in D$,

$\beta d=v(d) \beta$ and $(h+\beta) d=v(d)(h+\beta)$

$\left(\beta d=\rho^{-1} b d \stackrel{(38)}{=} \rho^{-1} \sigma \tau(d) b=\omega_{\rho^{-1}} \sigma \tau(d) \beta=v(d) \beta\right)$. If, in addition, we assume that the element $b$ is a left regular element $D$. Then $\sigma \tau=\omega_{\rho} v=\omega_{b}$ and the element $\beta \in D$ is also left regular in $D$. By (44), $\beta \beta=v(\beta) \beta$ and $(h+\beta) \beta=v(\beta)(h+\beta)$. Hence,

$\nu(\beta)=\beta, \quad h \beta=\beta h, \quad \sigma\left(h^{i}\right)=\rho_{i}^{v} \sum_{j=0}^{i} \beta_{i j} h^{j} \quad(i \geq 1)$ where $\rho_{i}^{v}:=\rho v(\rho) \cdots v^{i-1}(\rho), \beta_{i j}=\left(\begin{array}{c}i \\ j\end{array}\right) \beta^{i-j}$.

In more detail, $\sigma\left(h^{i}\right)=(\rho(h+\beta))^{i}=\rho_{i}^{\nu}(h+\beta)^{i}=\rho_{i}^{\nu} \sum_{j=0}^{i} \beta_{i j} h^{j}$.

Theorem 6.4 is a simplicity criterion for a diskew polynomial ring $E=D[x, y ; \sigma, \tau, b, \rho]$ where $\rho$ is a unit and $\tau \sigma$ is an epimorphism. By Theorem 6.2, the ring $E$ is a GWA that satisfies the assumptions of Theorem 4.2, a simplicity criterion for GWAs, and Theorem 6.4 is rather a straightforward corollary of Theorem 4.2 and (45).

Theorem 6.4 Let $E=D[x, y ; \sigma, \tau, b, \rho]$ be a diskew polynomial ring such that $\rho$ is a unit in $D$ and $v:=\tau \sigma$ is an epimorphism. The following statements are equivalent.

1. The ring $E$ is a simple ring.

2. (a) The endomorphisms $\sigma$ and $\tau$ of $D$ are automorphisms,

(b) the ring $D$ is a $\sigma$-simple ring,

(c) for each natural number $n \geq 1$ there is no element $p=h^{n}+\sum_{i=0}^{n-1} d_{i} h^{i} \in \mathcal{D}$, where $d_{i} \in D$, such that

(i) for all elements $d \in D, p d=v^{n}(d) p$, i.e., $d_{i} d=v^{n-i}(d) d_{i}$ for $i=0,1, \ldots, n-1$,

(ii) $\sigma(p)=\rho_{n}^{v} p$ where $\rho_{n}^{v}=\rho v(\rho) \cdots v^{n-1}(\rho)$, and

(iii) $[h, p]=0$, i.e, $v\left(d_{i}\right)=d_{i}$ for $i=0,1, \ldots, n-1$, and

(d) the elements $b_{i} \in D$ (see (40)), where $i \geq 1$, are units in $D$. In particular, $b \in D$ is a unit.

3. (a) The endomorphisms $\sigma$ and $\tau$ of $D$ are automorphisms,

(b) the ring $D$ is a $\tau$-simple ring,

(c) for each number $n \geq 1$ there is no element $p^{\prime}=h^{\prime n}+\sum_{i=0}^{n-1} d_{i}^{\prime} h^{\prime i} \in \mathcal{D}=D\left[h^{\prime}, \mu:=\sigma \tau\right]$, where $d_{i}^{\prime} \in D$ and $h^{\prime}=\sigma(h)$, such that

(i) for all elements $d \in D, p^{\prime} d=\mu^{n}(d) p^{\prime}$, i.e., $d_{i}^{\prime} d=\mu^{n-i}(d) d_{i}^{\prime}$ for $i=0,1, \ldots, n-1$,

(ii) $\tau\left(p^{\prime}\right)=\left(\rho^{-1}\right)_{n}^{\mu} p$ where $\left(\rho^{-1}\right)_{n}^{\mu}:=\rho^{-1} \mu\left(\rho^{-1}\right) \cdots \mu^{n-1}\left(\rho^{-1}\right)$, and 
(iii) $\left[h^{\prime}, p^{\prime}\right]=0$, i.e, $\mu\left(d_{i}^{\prime}\right)=d_{i}$ for $i=0,1, \ldots, n-1$, and

(d) the elements $b_{i}^{\prime} \in D$ (see (41)), where $i \geq 1$, are units in $D$. In particular, $b \in D$ is a unit.

We keep the notation and assumptions of Theorem 6.4. Let $p=h^{n}+\sum_{i=0}^{n-1} d_{i} h^{i} \in \mathcal{D}$. Since $\rho$ is a unit, then, by (45), the condition that $\sigma(p)=\rho_{n}^{v} p$ is equivalent to the equalities (since $\left.\sigma(p)=\rho_{n}^{v} h^{n}+\cdots\right)$

$\rho_{n-j}^{v} d_{j}-\sigma\left(d_{j}\right)=\left(\begin{array}{c}n \\ j\end{array}\right) b^{n-j}+\sum_{j<i \leq n-1}\left(\begin{array}{l}i \\ j\end{array}\right) \sigma\left(d_{i}\right) b^{i-j}$ for $j=0,1, \ldots, n-1$.

In particular, for $j=n-1$ and $j=0$ we have, respectively, the equalities

$$
\begin{aligned}
& \rho d_{n-1}-\sigma\left(d_{n-1}\right)=n b, \\
& \rho_{n}^{v} d_{0}-\sigma\left(d_{0}\right)=b^{n}+\sum_{1 \leq i \leq n-1} \sigma\left(d_{i}\right) b^{i} .
\end{aligned}
$$

Let $p=h^{n}+\sum_{i=0}^{n-1} d_{i} h^{i} \in \mathcal{D}$ be as in Theorem 6.4. Notice that the element $\beta$ is a unit, $v=\omega_{\rho^{-1} b}=\omega_{\beta}$ and $p \beta=\beta p$. Then, $p=\omega_{\beta}(p)=v(p)=\tau \sigma(p)=\tau\left(\rho_{n}^{v} p\right)$, and so

$\tau(p)=\tau\left(\rho_{n}^{v}\right)^{-1} p$.

The next theorems shed light on the elements $p$ and $p^{\prime}$ in Theorem 6.4. Theorem 6.5 describes the element $p$ in Theorem 6.4 where $n=1$; (46)-(48) are used in the proof.

Theorem 6.5 Let $E=D[x, y ; \sigma, \tau, b, \rho]$ be a diskew polynomial ring such that $\rho$ is a unit and $\mathcal{D}=D[h ; v=\tau \sigma]$ where $h=y x$. The following statements are equivalent.

1. There exists an element $C=h+\alpha \in \mathcal{D}$, where $\alpha \in D$, such that $C d=v(d) C$ for all elements $d \in D$ and $\sigma(C)=\rho C$.

2. There is an element $\alpha \in D$ such that $\rho \alpha-\sigma(\alpha)=b$ and $\alpha d=v(d) \alpha$ for all elements $d \in D$.

If one of the equivalent conditions holds then $[h, C]=(v(\alpha)-\alpha) C$ and

(a) $C=\rho^{-1}(x y+\sigma(\alpha)), x C=\rho C x$ and $y C=\tau\left(\rho^{-1}\right)(C+v(\alpha)-\alpha) y$.

(b) $E \simeq D[C ; \nu][x, y ; \sigma, \tau, a:=C-\alpha]$ is a GWA where $\sigma(C)=\rho C$ and $\tau(C)=\tau\left(\rho^{-1}\right)(C+v(\alpha)-\alpha)$. Furthermore, $\tau \sigma(C)=C+v(\alpha)-\alpha$ and $\sigma \tau(C)=\sigma \tau\left(\rho^{-1}\right)(\rho C+\sigma(\nu(\alpha)-\alpha))$.

The canonical left normal element $C$ of a diskew polynomial ring. Theorem 6.6 is a criterion for an element $C=h+\alpha($ where $\alpha \in D)$ to be a left normal element in $E$.

Theorem 6.6 Let $E=D[x, y ; \sigma, \tau, b, \rho]$ be a diskew polynomial ring such that $\rho$ is a unit, $\mathcal{D}=D[h ; v=\tau \sigma]$ and $C=h+\alpha$ where $h=y x$ and $\alpha \in D$. The following statements are equivalent.

1. The element $C$ is left normal in $E$.

2. $\rho \alpha-\sigma(\alpha)=b, v(\alpha)=\alpha$ and $\alpha d=v(d) \alpha$ for all elements $d \in D$.

If one of the equivalent conditions holds then $[h, C]=0$ and

(a) $C=\rho^{-1}(x y+\sigma(\alpha)), x C=\rho C x$ and $y C=\tau\left(\rho^{-1}\right) C y$.

(b) $E \simeq D[C ; \nu][x, y ; \sigma, \tau, a:=C-\alpha]$ is a GWA where $\sigma(C)=\rho C$ and $\tau(C)=\tau\left(\rho^{-1}\right) C$.

(c) The element $C$ is a left normal, left regular element of $E$ and $E /(C) \simeq D[x, y ; \sigma, \tau,-\alpha]$ is a GWA.

(d) The element $C$ is a normal element in $E$ iff $\operatorname{im}(v)=D$.

(e) The element $C$ is regular iff $C$ is right regular iff $\operatorname{ker}(v)=0$.

(f) The element $C$ is a normal, regular element iff $v$ is an automorphism of $D$.

The canonical central element $C$ of a diskew polynomial ring (under certain conditions) The next corollary is a criterion for an element $C+\alpha$ (where $\alpha \in D$ ) to be a central element in $E$. It follows straightaway from Theorem 6.6. 
Corollary 6.7 Let $E=D[x, y ; \sigma, \tau, b, \rho]$ be a diskew polynomial ring such that $\rho$ is a unit, $\mathcal{D}=D[h ; v=\tau \sigma]$ and $C=h+\alpha$ where $h=y x$ and $\alpha \in D$. The following statements are equivalent.

1. The element $C$ is a central element of $E$.

2. $\rho=1, v=1, \alpha-\sigma(\alpha)=b$, and the element $\alpha$ belongs to the centre of $D$.

If one of the equivalent conditions holds then

(a) $C=x y+\sigma(\alpha)$.

(b) $E \simeq D[C][x, y ; \sigma, \tau, a:=C-\alpha]$ is a GWA where $\sigma(C)=C$ and $\tau(C)=C$.

(c) The element $C$ is a regular element of $E$.

Every simple ring is, in fact, an algebra either over the field of rational numbers $\mathbb{Q}$ or over the finite prime field $\mathbb{F}_{p}$ that contains $p$ elements ( $p$ is a prime number).

Simplicity criterion for DPRs in characteristic zero If the ring $D$ is a $\mathbb{Q}$-algebra the condition (c) in Theorem 6.4 can be replaced by condition 4 of Theorem 6.6.

Theorem 6.8 Let $E=D[x, y ; \sigma, \tau, b, \rho]$ be a diskew polynomial ring such that $\rho$ is a unit in $D, v:=\tau \sigma$ is an epimorphism and $D$ is a $\mathbb{Q}$-algebra. The following statements are equivalent.

1. The ring $E$ is a simple ring.

2. (a) The endomorphisms $\sigma$ and $\tau$ of $D$ are automorphisms,

(b) the ring $D$ is a $\sigma$-simple ring,

(c) there is no element $\alpha \in D$ such that $\rho \alpha-\sigma(\alpha)=b$ and $\alpha d=v(d) \alpha$ for all elements $d \in D$, and

(d) the elements $b_{i} \in D$ (see (40)), where $i \geq 1$, are units in $D$. In particular, $b \in D$ is a unit.

Simplicity criterion for DPRs in prime characteristic $p$ If the ring $D$ is a $\mathbb{F}_{p}$-algebra the condition (c) in Theorem 6.4 can be replaced by more explicit conditions (where $\mathbb{F}_{p}=\mathbb{Z} / p \mathbb{Z}$ ).

Theorem 6.9 Let $E=D[x, y ; \sigma, \tau, b, \rho]$ be a diskew polynomial ring such that $\rho$ is a unit in $D, v=\tau \sigma$ is an epimorphism and $D$ is a $\mathbb{F}_{p}$-algebra. The following statements are equivalent.

1. The ring $E$ is a simple ring.

2. (a) The endomorphisms $\sigma$ and $\tau$ of $D$ are automorphisms,

(b) the ring $D$ is a $\sigma$-simple ring,

(c) for each natural number $n \geq 0$ there is no element $p^{\prime}=h^{p^{n}}+\sum_{i=0}^{n-1} \alpha_{i} h^{p^{i}}+\alpha$, where $\alpha, \alpha_{i} \in D$, such that

(i) for all $d \in D, p d=v^{p^{n}}(d) p$, i.e. $\alpha d=v^{p^{n}}(d) \alpha$ and $\alpha_{i} d=v^{p^{n}-p^{i}}(d) \alpha_{i}$ for $i=0,1, \ldots, n-1$,

(ii) $\sigma\left(p^{\prime}\right)=\rho_{p^{n}}^{v} p^{\prime}$,

(iii) $\left[h, p^{\prime}\right]=0$, i.e. $v(\alpha)=\alpha$ and $v\left(\alpha_{i}\right)=\alpha_{i}$ for $i=0,1, \ldots, n-1$.

(d) the elements $b_{i} \in D$ (see (40)), where $i \geq 1$, are units in $D$. In particular, $b \in D$ is a unit.

3. (a) The endomorphisms $\sigma$ and $\tau$ of $D$ are automorphisms,

(b) the ring $D$ is a $\sigma$-simple ring,

(c) there is no element $\alpha \in D$ such that $\rho \alpha-\sigma(\alpha)=b, v(\alpha)=\alpha$ and $\alpha d=\nu(d) \alpha$ for all elements $d \in D$, and for each natural number $n \geq 1$ there are no elements $\alpha, \alpha_{0}, \ldots, \alpha_{n}$ such that

(i) for all $d \in D, \alpha d=v^{p^{n}}(d) \alpha$ and $\alpha_{i} d=v^{p^{n}-p^{i}}(d) \alpha_{i}$ for $i=0,1, \ldots, n-1$,

(ii) $\sigma\left(\alpha_{i}\right)=\rho_{p^{n}-p^{i}}^{v} \alpha_{i}$ for $i=0,1, \ldots, n-1$, and $\rho_{p^{n}}^{v} \alpha-\sigma(\alpha)=b^{p^{n}}+\sum_{i=0}^{n-1} \sigma\left(\alpha_{i}\right) b^{p^{i}}$,

(iii) $v(\alpha)=\alpha$ and $v\left(\alpha_{i}\right)=\alpha_{i}$ for $i=0,1, \ldots, n-1$.

(d) the elements $b_{i} \in D$ (see (40)), where $i \geq 1$, are units in $D$. In particular, $b \in D$ is a unit.

Open Access This article is distributed under the terms of the Creative Commons Attribution 4.0 International License (http:// creativecommons.org/licenses/by/4.0/), which permits unrestricted use, distribution, and reproduction in any medium, provided you give appropriate credit to the original author(s) and the source, provide a link to the Creative Commons license, and indicate if changes were made. 


\section{References}

1. Bavula, V.V.: Finite-dimensionality of $\mathrm{Ext}^{n}$ and $\mathrm{Tor}_{n}$ of simple modules over a class of algebras. Funct. Anal. Appl. 25(3), 229-230 (1991)

2. Bavula, V.V.: Classification of simple sl(2)-modules and the finite-dimensionality of the module of extensions of simple sl(2)modules. Ukr. Math. J. 42(9), 1044-1049 (1990)

3. Bavula, V.V.: Simple $D[X, Y ; \sigma, a]$-modules. Ukr. Math. J. 44(12), 1500-1511 (1992)

4. Bavula, V. V.: Generalized Weyl algebras and their representations. (Russian) Algebra i Analiz 4(1), 75-97 (1992); translation in St. Petersburg Math. J.4(1), 71-92 (1993)

5. Bavula, V.V.: Generalized Weyl algebras, kernel and tensor-simple algebras, their simple modules. In: Representations of Algebras (Ottawa, ON, 1992), Proceedings of CMS Conference, American Mathematical Society, Providence, RI, vol. 14, pp. 83-107 (1993)

6. Bavula, V.V.: Extreme modules over the Weyl algebra $A_{n}$. Ukr. Math. J. 45(9), 1327-1338 (1993)

7. Bavula, V.V.: Description of two-sided ideals in a class of noncommutative rings. I. Ukr. Math. J. 45(2), 223-234 (1993)

8. Bavula, V.V.: Description of two-sided ideals in a class of noncommutative rings. II. Ukr. Math. J. 45(3), 329-334 (1993)

9. Bavula, V.V.: Filter dimension of algebras and modules, a simplicity criterion of generalized Weyl algebras. Commun. Algebra 24(6), 1971-1992 (1996)

10. Bavula, V. V.: Generalized Weyl algebras and diskew polynomial rings. https://arxiv.org/abs/1612.08941

11. McConnell, J.C., Robson, J.C.: Noncommutative Noetherian Rings. Wiley, Chichester (1987)

12. Zachos, C.: Elementary paradigms of quantum algebras. Contemporary Math. 134, 351-377 (1992) 